\title{
Multi-user Synchronisation in ad hoc OFDM-based Wireless Personal Area Networks
}

\author{
VÍCTOR P. GIL JIMÉNEZ and ANA GARCÍA ARMADA \\ Dept. Signal Theory and Communications, University Carlos III de Madrid, 28911 Leganés, Madrid, Spain \\ E-mails: \{vgil,agarcia\}@tsc.uc3m.es
}

\begin{abstract}
In this paper, a procedure for dealing with the multi-user synchronisation problem in Orthogonal Frequency Division Multiple Access (OFDMA)-based systems for ad hoc environments is proposed and analysed. We show with this novel approach that it is possible to re-use much of the already extent literature for single-user synchronisation in OFDM and apply it to multi-user ad hoc scenarios. Also a distributed version of the procedure is proposed in order to fairly share out the power consumption among all the devices. The proposed procedure makes use of higher layer capabilities in a cross-layer design and it does not incur too much complexity or power. This issue is specially critical in wireless heterogeneous ad hoc networks where devices can be very limited in terms of transmission and/or computational power.
\end{abstract}

Keywords: OFDMA, H-OFDM, ad hoc networks, multi-user synchronisation

\section{Introduction}

Synchronisation is an important task in Orthogonal Frequency Division Multiplexing (OFDM) and Orthogonal Frequency Division Multiple Access (OFDMA) as it is shown in many references such as [1-3]. For that reason, the literature is very extent on this topic for the earlier single-user OFDM case starting from the origins in OFDM synchronisation [4 or 5] to recent references such as [6 or 7]. However, for the multi-user scenario there are still only a few proposals [8] and these contributions are generally suitable for infrastructure-based environments, i.e. there is a Base Station (BS) or central node that is able to coordinate transmission. Proposals for ad hoc networks are mainly applicable to Time Division Multiple Access (TDMA) systems $[9,10]$.

In an OFDMA-based system, all the devices simultaneously involved in the transmission share the same time but different orthogonal frequencies and therefore they must be synchronised to each other in order to avoid the so-called Multi-User Interference (MUI). For this purpose, one of the terminals must establish the time and frequency reference for every equipment and the other ones should synchronise to it. In Wireless Local Area Networks (WLAN) two different kinds of environments can be distinguished, namely, Infrastructure-Based and ad hoc, whereas in Wireless Personal Area Networks (WPAN) only the ad hoc scenario is suitable. In the firs one, a BS or similar entity (Access Point, Node Coordinator, etc.) is always present. This BS acts, among other things, as the time and frequency reference and all the mobile terminals use the beacon signals or preambles sent by this BS for the synchronisation task [8]. On the other hand, in ad hoc environments there is no such a central coordinating 
entity, and therefore one of the terminals in the network must establish the time and frequency reference. Besides, since we are dealing with wireless devices, the power consumption has to be handlled very carefully, i.e. no one would like that the life of his terminal battery becomes much shorter because the device has taken the role of the master for synchronisation. For this reason, in this paper a leader procedure is proposed in order to establish a mechanism for dealing with the problem of multi-user OFDMA synchronisation while saving power. This leader will be the terminal establishing the time and frequency reference for the entire personal/local area network. The aim of the procedure is to allow the re-use of some of the strategies in the literature for the single-user synchronisation to the multi-user environment by reducing the multi-user problem to a single-user one with application to the ad hoc scenario. To this end higher layer capabilities are used. We will examine the performance of the proposed scheme for a combination of OFDMA and TDMA define for WPAN systems. Besides, a distributed version of the procedure is also proposed in order to share out the power consumption among all the devices.

The remaining of the paper is organised as follows. In Sections 2 and 3, the description of the system and the single-user OFDM synchronisation are presented. In Section 4, the proposed synchronisation procedure called leader procedure is described jointly with a distributed version. The results for the proposed procedure are shown in Section 5 and finall, in Section 6 some conclusions are given.

\section{System Description}

In this section, the multi-user OFDM signal will be described. Special attention will be paid to the Hybrid-OFDM (H-OFDM) signal structure define for WPAN scenarios in the frame of PACWOMAN project [11], that is going to be used to illustrate the performance of the proposed synchronisation scheme.

\subsection{Multi-User OFDM Signal}

In an perfectly synchronised OFDMA system, where several users are continuously transmitting their information, the composite discrete-time transmitted signal can be written as:

$$
s^{\ell}[n]=\sum_{\ell=0}^{\infty} \sum_{k=0}^{N-1} X_{k}^{\ell} e^{j \frac{2 \pi}{N} k(n-\ell N)},
$$

where $n, k$ and $\ell$ denote the sample index, the sub-carrier index and OFDM symbol index, respectively, $N$ is the number of sub-carriers and $X_{k}^{\ell}$ are the complex data sent by different users. In a pure OFDMA system such as IEEE 802.16a [12], a group of sub-carriers is assigned to a user during the whole transmission until it finishes i.e. a user is represented by a set of sub-carriers ( $k \in \kappa_{u}$, where $\kappa_{u}$ is the set of sub-carriers for $u$ th user).

\subsection{H-OFDM Multi-User System}

The leader procedure has been proposed within PACWOMAN project [11]. The system is a TDMA/OFDMA combination called H-OFDM. The transmission is organised in frames, each frame is divided into 16 slots and every slot can allocate up to 150 OFDM symbols. These 
Table 1. Main H-OFDM parameters

\begin{tabular}{ll}
\hline Parameter & Value \\
\hline Number of sub-carriers $(N)$ & 64 \\
Number and distribution of frequency guards & Eight at each boundary \\
Number and distribution of pilots & Full-pilot preamble and four scattered in every symbol \\
Number of useful sub-carriers & 44 \\
Bandwidth & $10 \mathrm{MHz}$ \\
Duration of the Cyclic Prefi & $400 \mathrm{~ns}$ \\
Duration of the useful OFDM symbol & $6.4 \mu \mathrm{s}$. \\
Number of slots per frame & 16 \\
Slot length & $1.05 \mu \mathrm{s} .\left(N_{\mathrm{OFDM}}=150\right)$ \\
$R_{\mathrm{b}}$ (Data Rate) & $9.2 \mathrm{Kbps}-38.8 \mathrm{Mbps}$ \\
\hline
\end{tabular}

symbols are shared by different users, i.e. it is a packet-based OFDMA system. In this case, in Equation (1), a user is allocated the pair number of sub-carrier and OFDM symbol, i.e. $k \in \kappa_{u}$ and $l \in \iota_{u}$, where $\iota_{u}$ is the set of OFDM symbols assigned to $u$ th user.

The main system parameters are shown in Table 1 [13]. Each packet has a header of fi e OFDM symbols devoted to acquisition, channel estimation and synchronisation/tracking. The structure of the header is similar to other packet-based systems such as IEEE 802.11a [14] with a structure divided in three parts, the firs one has two equal symbols, the second one has two other identical symbols and the last symbol is reserved for information about the packet characteristics. By using the header it is possible for the users to get synchronised in pairs applying algorithms already proposed in the literature [5 or 7]. However, all the devices in the network have to be synchronised to each other in order to avoid the MUI.

\subsection{CONTROL Cha NnEls}

In PACWOMAN, the firs slot of every frame, denoted as Control Slot (CS), is reserved for control purposes. It is mandatory that every equipment in the network listens to this control slot. Also, this slot is divided into three different physical channels, namely, Paging and Access Grant Channel (PAGCH), Resources Access Channel (RACH) and Leader Channel (LCH) as it can be seen in Figure 1. The PAGCH is used by terminals for requesting/responding to a communication initiation attempt. All the devices inform the others in the RACH about resources to be used/released by them. In this way all the terminals perceive the whole picture about free/busy resources in the network. A resource here is given by the pair: number of subcarrier, number of slot $(k, l)$ [15]. The LCH is used by the leader for sending synchronisation messages to the terminals. We will mainly focus on $\mathrm{LCH}$ channel in this paper.

It should be noted that the leader procedure has been proposed for PACWOMAN system but it can be easily applied to any other system where all the devices listen to a special channel that may therefore be used for synchronisation tasks. 


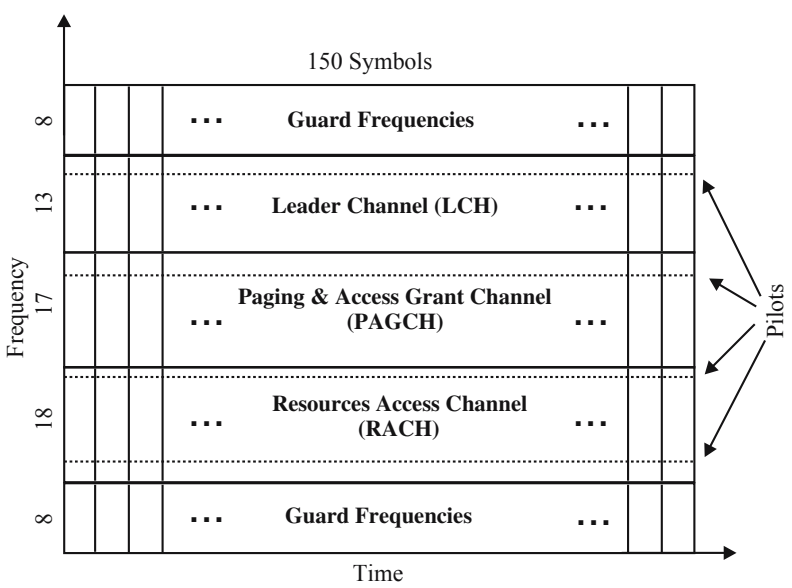

Figure 1. Control slot structure.

\section{Single-User OFDM Synchronisation}

Synchronisation is a critical task in any OFDM receiver since the global system performance will be determined by the accuracy in the synchronisation process. The problem for the single-user scenario has been extensively analysed. Most of the proposals lean on Schmidl and Cox ideas [5]: the transmission of two or more identical sequences (e.g. in the packet header) that are correlated in the receiver. The way the correlation is performed, the number of equal sequences, the data conveyed in these sequences or the metric used for detecting packets vary from one approach to another. In order to firstl detect the start of the OFDM symbol a metric is defined This metric is usually normalised by the energy of the received signal and/or weighted. When the metric reaches its maximum value the packet is acquired. When implementing the system this metric has to be estimated by using peak detectors [16]. The metric proposed in [5] is:

$$
M[n]=\frac{|P[n]|^{2}}{(R[n])^{2}}
$$

being $P[n]$ the correlation function:

$$
P[n]=\sum_{m=0}^{L-1}\left(r^{*}[n+m] r[n+m+L]\right),
$$

where $r$ is the time-domain discrete received signal, $L$ the size of the window over which the correlation is performed and ${ }^{*}$ denotes the conjugate operation. As it has been mentioned, the correlation is usually normalised by the received energy, in this case $R[n]$ :

$$
R[n]=\sum_{m=0}^{L-1}|r[n+m+L]|^{2} .
$$


Regarding the frequency offset estimation, algorithms make use of the previous already calculated correlation. In [5], frequency offset is obtained as:

$$
\hat{\Delta} f=\frac{\angle(P[n])}{\pi T}
$$

with $P[n]$ evaluated at the value of $n$ which maximises the metric. $T$ is the OFDM symbol period and $\angle$ denotes the angle function.

In order to reduce the variance in the estimation of the start of the packet and the frequency offset several improvements to these ideas have been proposed. It is shown in [17] that for a given packet preamble length, the more number of identical sub-parts in the preamble the better performance. Besides, in [18] the use of hermitic sub-parts is explored. In [7], based on those ideas and others from the authors [19], they proposed a two stage synchronisation: coarse and fine with a preamble divided into $N_{L}$ sub-parts weighted by a sign pattern. For the coarse stage the preamble is used, then channel is estimated and the synchronisation is refine using the channel estimation in the fin stage. Those ideas from [7] have been taken for the peer-to-peer synchronisation part with small variations in our leader procedure.

\section{The Leader Procedure}

In this section, an explanation on the leader procedure will be provided. As it has been already mentioned, all the terminals in the network must be synchronised to each other. Thus the firs action for a new incoming terminal is getting synchronised. The synchronisation process is divided into two parts, namely, coarse synchronisation followed by fin synchronisation. Agorithms achiving both steps in the synchronisation process will be explained in Section 4.2 in more detail. For the design of the leader procedure higher layer capabilities, such as Medium Access Control (MAC) layer, are proposed to be used together with Physical layer (PHY). Involving several layers into the procedure, what is called cross-layer design, some improvements can be obtained. In our case, the PHY design is simplifie due to the complexity reduction in the synchronisation process. As for the MAC layer in ad hoc network access the busy-tone or dual busy-tone [20,21] has been proposed: when a terminal is willing to transmit it activates a frequency tone in order to avoid hidden-terminal problems [22], but from the point of view of the PHY, it only requests for a MAC procedure independently on how it works. Once the hidden terminal problems have been readily avoided by the MAC procedures, for the case of synchronisation, and the other transmission procedures (establishment, released, etc.) not only a tone but a control slot is also needed in order to transmit some additional important information, i.e. we need a control slot.

In the following, the description of the leader procedure and a theoretical model for its performance analysis will be provided. Besides, the algorithms proposed for synchronisation will be described and a distributed version of the procedure will be also stated.

\subsection{DESCRIPTION OF THE Procedure}

The state diagram for this procedure is depicted in Figure 2. When a terminal is switched-on to the network, firs it will look for the leader by sensing the channel and checking whether some other equipment is transmitting or not. If the channel is busy, the terminal can easily get the coarse synchronisation as will be explained later in Section 4.2. However, even though 


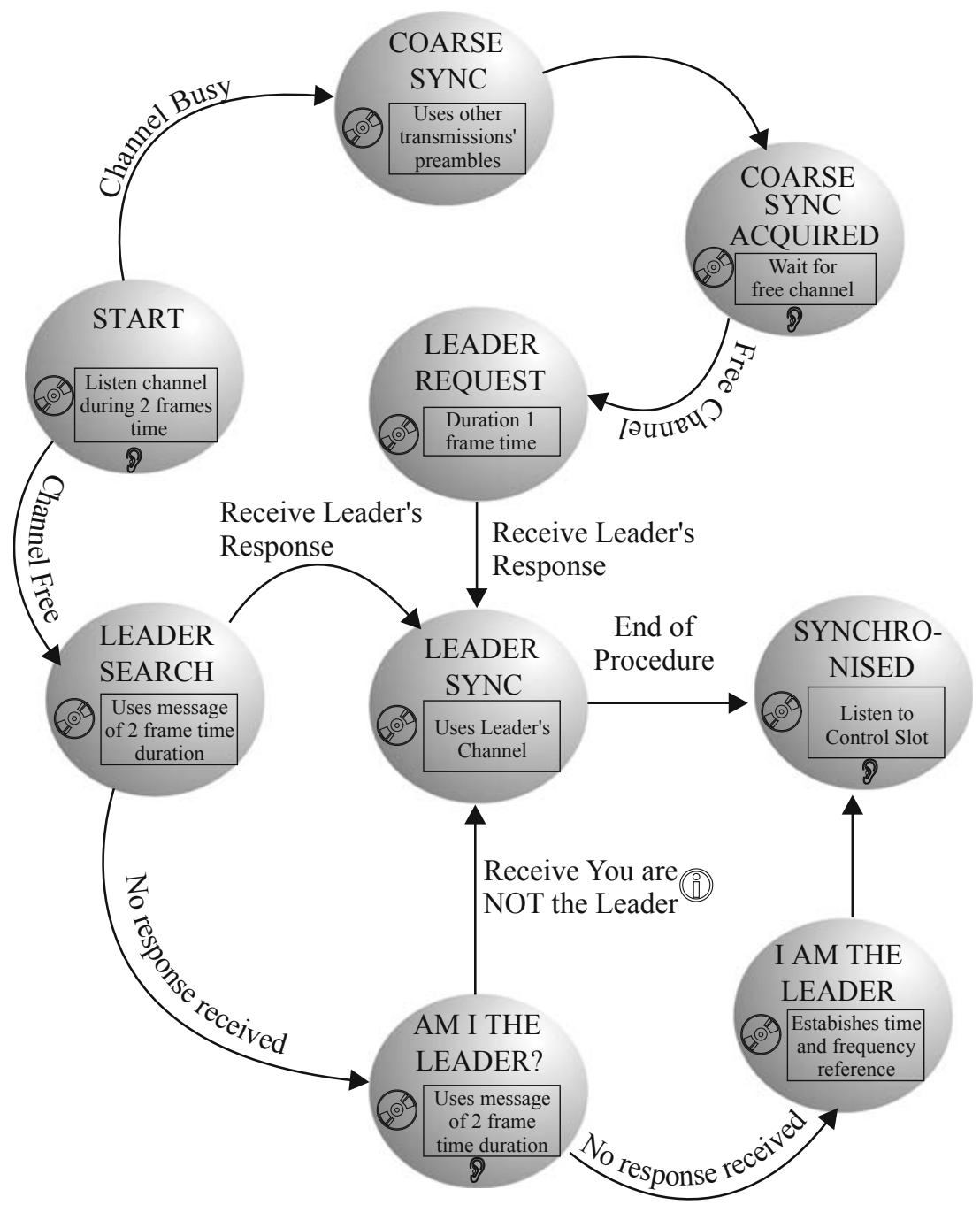

Figure 2. The leader procedure state diagram.

the time and frequency reference can be obtained accurately enough it does still not know where the firs slot is located, i.e. the terminal has only a relative time reference. In order to get the absolute time reference and more precision in the frequency and time estimates, once the channel is free it must send a request message looking for the leader, i.e. request for leader message. The leader will answer by using the LCH (and therefore the new terminal will determine the absolute time reference) and the new device will achieve fin synchronisation with the leader. The new already synchronised equipment must keep listening to the CS and tracking for synchronisation when other devices would use this CS. In this way the time and frequency references are tracked and therefore deviations will be kept down to a reasonable margin. However, it is suggested that if there are no transmissions for a long-time the next terminal transmitting should firs request a new synchronisation in order to refresh it; this is not only useful for the terminal itself but also for the others. 
On the other hand, if no one is transmitting the new terminal has no choice for getting synchronised. It may be because there is no other terminal (and so, the incoming device will take the role of the leader for synchronisation, establishing the time and frequency references), or it is because the other terminals are silent. In this situation, the terminal firs listens to the channel for two frames-duration (because the new device does not know anything about the time reference it has to listen at least for a period of more than one frame), and sends a leader request also during two frames-time (again for the same reason, it has to be guarantied that the message interferes at least one control slot in order to be listened by the leader). The leader terminal will listen to requests and it will answer analogously as in the previous situation allowing the incoming device to get synchronisation with the leader (coarse synchronisation by using the header in the packet and fin by using the transmission in the LCH). The state diagram for the leader procedure with the possible situations is shown in Figure 2 where each bubble represents a state and boxes indicate the action(s) taken at this state.

Regarding the power consumption, the terminal assuming the leader role does not expend much more energy than the other terminals: It should listen to the control slot as all the devices in the network should and it only has to send the synchronisation responses for fin synchronisation when prompted.

\subsection{AlgorithMS FOR SYNCHRONISATION}

The following sections describe the proposed algorithms to achieve coarse and fin synchronisation.

\subsubsection{Coarse synchronisation}

Each packet has a header composed by fi e OFDM symbols. The two firs symbols are devoted to acquisition, Automatic Gain Control (AGC) and synchronisation. The proposed preamble and algorithm for the coarse synchronisation are based on a slight modificatio of [7]. The preamble is divided into $N_{L}=8 \mathrm{sub}$-parts and the sequence pattern is $p_{s}=[++-+++-+] .{ }^{1}$ And the synchronisation is acquired when the metric $M_{\text {prop }}$ reaches the maximum:

$$
M_{\text {prop }}[n]=\left(\frac{N_{L}}{N_{L}-1} \frac{\left|P_{\text {prop }}[n]\right|}{R_{\text {prop }}[n]}\right)^{2},
$$

where

$$
P_{\text {prop }}[n]=\sum_{\ell=0}^{N_{L}-2} p_{S}[\ell] p_{S}[\ell+1] \cdot \sum_{m=0}^{N / N_{L}-1} r^{*}\left[n+\ell \frac{N}{N_{L}}+m\right] S_{S}\left[n+(\ell+1) \frac{N}{N_{L}}+m\right] \text { (7) }
$$

and

$$
R_{\text {prop }}[n]=\sum_{m=0}^{N / N_{L}-1} \sum_{\ell=0}^{N_{L}-1}\left|r\left[n+m+\ell \frac{N}{N_{L}}\right]\right|^{2}
$$

The difference with respect to [7] lies in the correlation $P_{\text {prop }}[n]$ (Equation 7) that is performed with the original sequence $S_{S}$ instead of the received one, in order to reduce the MUI. Besides, the sign pattern is also slightly different. As in other proposals, this correlation is normalised with respect to the mean energy of the signal, i.e. $R_{\text {prop }}[n]$. Regarding the frequency

\footnotetext{
1 The signs represent whether the original short sequence to be used in each sub-part is inverted or not.
} 
offset estimation, it is obtained as in [7] by substracting the phases of the correlation functions of each part of the preamble.

\subsubsection{Fine synchronisation}

In order to improve the results obtained with coarse synchronisation, once this firs stage is acquired the fin synchronisation process is performed for further tuning. This process is based on [6] and [7] and it is summarised as follows. By using the other two training sequences in the preamble, an estimate of the channel can be obtained. Besides, in the LCH there is another training sequence specially inserted with the purpose of improving the estimation. By using these estimates the channel taps are obtained and the timing acquisition is adjusted to the strongest tap. Then, the time and frequency synchronisation are performed again in order to obtain better results. Moreover, since this special message is only sent in the LCH and it is allocated into the CS, the absolute time reference is also acquired in the fin stage.

\subsection{ThEORETICAL ModeL}

In order to give some insight about performance in terms of time spent for synchronisation without restorting to simulations the system can be approximately modelled as a queuing system M/M/1 (following Kendall notation) [23]. This model is motivated by the fact that there is a common resource, i.e. the control slot, arrivals can be approximated as Poisson (as suggested for a firs approach regarding traffi in local networks in [24]) and time for service as exponential. Therefore, the mean time required for synchronisation can be computed as:

$$
W=\frac{E(s)}{1-\lambda E(s)},
$$

where $E(s)$ is the mean time for synchronisation based on the time that takes sending messages and receiving responses and $\lambda$ is the mean time between arrivals (synchronisation requests from terminals). The behaviour of this expression is exponentially increasing depending on the arrivals rate as it can be seen in Figure 3. Therefore if the number of terminals switching-on at the same time is large the synchronisation can be very slow. However, for a typical number of devices in a wireless network the values for synchronisation time are low enough. Besides, as it will be seen in the results section, the simulated behavior is not exponential due mainly to the multi-synchronisation or simultaneous synchronisation effect. Therefore, $M / M / 1$ model is only valid for a firs approach; a more precise performance evaluation of the leader procedure requires exhaustive simulations. The simultaneous synchronisation effect can be explained as follows: when the channel is busy and a device is coarsely synchronised by using preambles in the header, it must wait until the $\mathrm{LCH}$ is free in order to send the request for the leader message. If meanwhile another device tries to synchronise and it is also waiting for the free $\mathrm{LCH}$, once one of them sends the request for the leader message both of them (and all devices in the queue) can synchronise with that leader response.

\subsection{Distrib Uted Lea Der Procedure}

It has been shown that the leader does not spend a substantially higher energy when compared to the other terminals. Basically, the leader transmits more power than the others when responding. In order to fairly share out the energy consumed by the leader, a distributed leader procedure is also proposed. In this variation, instead of burdening only one terminal with the 


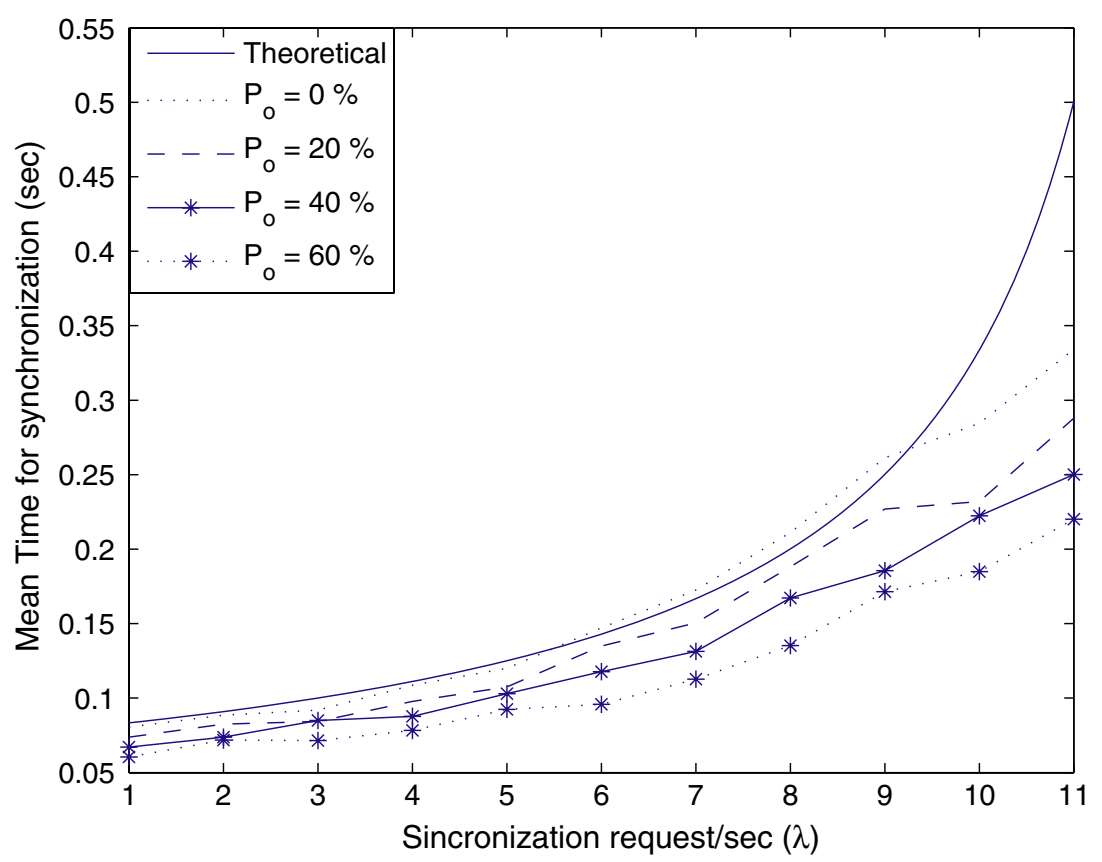

Figure 3. Mean time for synchronisation.

leader role, this role is alternately assumed by all the devices in the network. According to this new procedure when a terminal is switched-on it proceeds similarly as before, during the firs state. The only difference is that it must request for a specifi leader number when it sends the request for leader message. This number, $l_{n}$, is randomly generated in the range, e.g. from 0 to 255. The leader owning this number must respond. If no leader responds to the terminal, it assumes the role of the leader. Hence the only changes in the procedure are in the firs state (a specifi leader is requested), in the state when the device takes on the leadership, where the terminal assumes that it owns all the available numbers, and finall when the leader is synchronised to a new terminal, where it transfers to it half of the numbers that it holds. By applying this process recursively the leader role is distributed among all the terminals in the network and the extra power consumption is fairly shared out among all transceivers. Besides, the distributed leader procedure is more robust against conectivity errors.

\section{Results and Discussion}

In order to scrutinise the performance of the synchronisation process, Monte-Carlo simulations have been carried out in a scenario representing a PACWOMAN system where the main parameters were: HiperLAN 2 A channel model; 10 active users requesting synchronisation according to a Poisson distribution for arrivals; signal to noise ratio ranging from 15 to $25 \mathrm{~dB}$. Results have been obtained averaging over the whole range of SNR described above.

In Figure 3, the mean time to aquire synchronisation is shown depending on the number of terminals requesting the leader. Results are shown for different probabilities of channel occupation $\left(P_{o}\right)$ that represent the network's load; this is an important parameter from the synchronisation point of view as it will appear later. It can be observed that the behaviour of the 

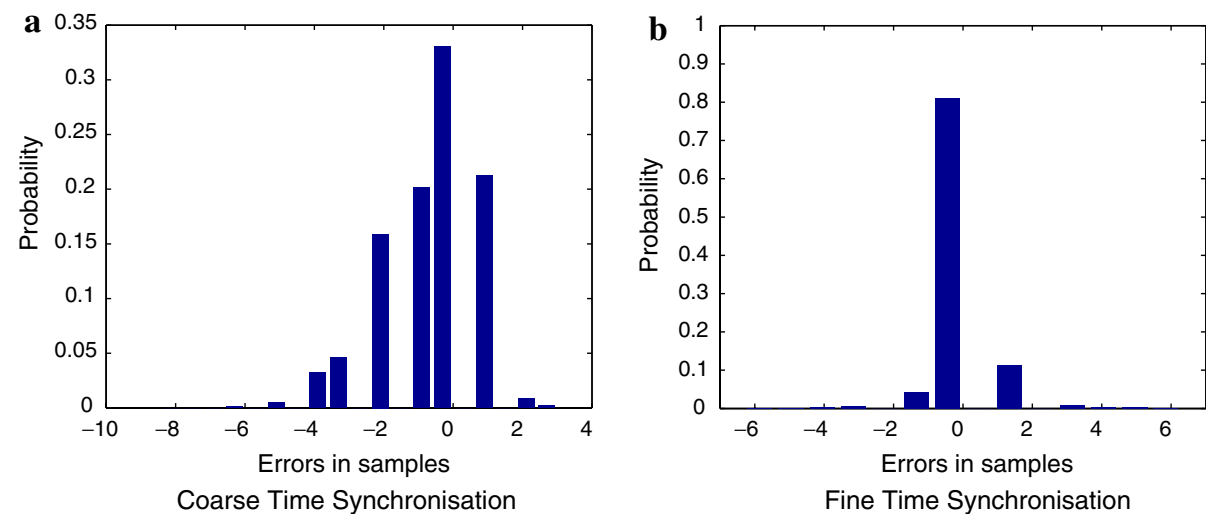

Figure 4. Histogram for time synchronisation.

curves is quite similar to the results obtained with the theoretical model (Section 4) for lower arrivals rate. However, if the number of requests increases the curve comes closer to linear that to the theoretically predicted exponential behaviour. This is a good property, because in this way the time for synchronisation keeps low even for large number of requests. The reason was mentioned before, there exists the possibility of multiple simultaneous synchronisations. This situation is more noticeable as the number of requests increases because the probability of two or more devices waiting for a free $\mathrm{LCH}$ increases as the number of terminal arrivals increases. Besides, the network occupation is also important because the higher load the lower time for synchronisation again due to simultaneous synchronisations using the preambles in the transmitted signals. According to these results the procedure can be easily scaled for more dense networks as for example sensor networks.

On the other hand, in Figure 4, histograms for time errors resulting from the synchronisation process are shown. In Figure 4a, solely coarse synchronisation is considered while both coarse and fin synchronisation are performed to obtain the histogram of Figure $4 \mathrm{~b}$. In both figures zero errors in samples means perfect synchronisation whereas positive errors occur when the algorithm determines that the start of the packet is delayed and negative ones when the synchronisation is acquired in advance. It can be seen that errors in time in Figure 4a are small but there still exists a variance in the results (in the figur this variance is about 2.3 samples). In order to reduce the variance, the fin synchronisation is applied, and it can be seen that the variance of results has been drastically reduced because of the close loop synchronisation based on the channel estimation. Even in frequency-selective channels results are quite accurate. In terms of normalised frequency offset estimation, the mean error is below $10^{-5}$, i.e $f_{\mathrm{e}} / \Delta f<10^{-5}$, where $\Delta f$ is the frequency spacing between sub-carriers and $f_{\mathrm{e}}$ is the frequency error.

\section{Conclusions}

In this paper, a mulit-user synchronisation procedure for OFDM-based ad hoc systems has been proposed and analysed. The procedure reduces the multi-user problem resulting from the ad hoc environment to a single-user problem and allows the re-use of many of the OFDM already exiting algorithms for the OFDMA case. Since single-user synchronisation for OFDM 
is a mature topic, there exists a wide literature and it is very convenient to have the possibility of re-using those insights. The algorithm here proposed makes use of a combination of two schemes available in the literature (slightly modified) however, any other new single-user synchronisation schemes that may arise can be applied too. The procedure makes joint use of PHY and MAC layers leveraging the capabilities of cross-layer design. Also a distributed version of the procedure is proposed in order to fairly share out energy expenditure among all the devices in the network. Besides, the performance in terms of time for acquiring synchronisation has been evaluated by simulation and results show that a good performance for a wireless personal/local area network environment can be reached. Moreover it can be easily scaled for systems with higher number of users such as sensor networks.

\section{Acknowledgements}

This work has been partially funded by European Commission (IST-2001-34157), Spanish Government (TIC2002-03498, TEC 2005-07477), Madrid Community (UC3M-TEC-05-027) and European Social Fund by a grant to the firs author.

\section{References}

1. M. Moeneclaey, "The Effect of Synchronization Errors on the Performance of Orthogonal Frequency Division Multiplexed (OFDM) Systems”, Proc. of COST 254 (Emergent Techniques for Communication Terminals), July 1997.

2. P. Robertson and S. Kaiser, "The Effects of Doppler spreads in OFDM(a) Mobile Radio Systems", Proc. IEEE Vehicular Technology Conference VTC Fall, Vol. 1, pp. 329-333, 1999.

3. A.G. Armada, V.P. G. Jiménez, and J. Darriba, "Analysis of Phase Noise Effects in Multi-User OFDM", Proc. IEEE First Internatinal Symposium on Control Communications, and Signal Processing (ISCCSP), March 2004.

4. P. Moose, "A Technique for orthogonal Frequency Division Multiplexing Frequency Offset Correction", IEEE Transactions on Communications, Vol. 42, No. 10, pp. 2908-2914, 1994.

5. T.M. Schmidl and D.C. Cox, "Robust frequency and timing synchronization for OFDM", IEEE Transactions on Communications, Vol. 45, No. 12, pp. 1613-1621, 1997.

6. B. Yang, K.B. Letaief, and R.S.C.Z. Cao, "Timing Recovery for OFDM transmission", IEEE Journal on Selected Areas in Communications (JSAC), Vol. 18, No. 11, pp. 2278-2291, 2000.

7. H. Minn, V. Bhargava, and K. Letaief, "A Robust Timing and Frequency Synchronization for OFDM Systems," IEEE Transactions on Wireless Comunications, Vol. 2, No. 4, pp. 822-839, 2003.

8. J.-J. de Beek, P.O. Borjesson, M.L. Boucheret, D. Landstrom, J.M. Arenas, P. Odling, C. Ostberg, M. Wahlqvist, and S.K. Wilson, "A Time and Frequency Synchronization Scheme for Multiuser OFDM", IEEE Journal on Selected Areas in Communications (JSAC), Vol. 17, No. 11, pp. 1900-1914, 1999.

9. D. Zhou and T.-H. Lai, "Analysis and Implementation of Scalable Clock Synchronization Protocols in IEEE 802.11 ad hoc Networks", Proc. of IEEE International Conference on Mobile Ad-hoc and Sensor Systems, Fort Lauderdale, (Florida), Vol. 1, pp. 255-263, 25-27 October 2004.

10. J. Kleider and S. Gifford, "Synchronization for Broadband OFDM Mobile ad hoc Networking: Simulation and Implementation", Proc. IEEE International Conference on Acoustics, Speech, and Signal Processing (ICASSP), Vol. 4, pp. 3756-3759, 2002.

11. "PACWOMAN: Power Aware Communication for Wireless OptiMised personal Area Networks (IST-200134157), Proposal", 2001. (www.imec.be/pacwoman)

12. "IEEE 802.16a: Air interface for fi ed broadband wireless access systems. amendment 2: MAC modification and additional Physical Layer specification for 2-11 GHz", ISBN 0-7381-4069-4, IEEE Standard, 2003. 
13. A.G. Armada, V.P.G. Jiménez, M.J. Fernández-Getino, and J.L. García, "H-OFDM Design for Wireless Personal Area Communications", Proc. IST Mobile Summit, 2003.

14. "WLAN Medium Access Control (MAC) and Physical Layer (PHY) specifications High-speed physical layer in the 5 GHz Band", ISBN 0-7381-2695-0, IEEE Standard, No. 802.11a, June 2001.

15. V.P.G. Jiménez and A.G. Armada, "Bit-loaded H-OFDM to Increase Capacity in WLAN/WPAN", Proc. IEEE Vehicular Technology Conference VTC Fall 04. Los Angeles., Vol. 1, No. 1, September 2004.

16. V.P.G. Jiménez, M.J.F.-G. García, F.J.G. Serrano, and A. G. Armada, "Design and Implementation of Synchronization and AGC for OFDM-based WLAN Receivers", IEEE Transactions on Consumer Electronics, Vol. 50, No. 4, pp. 1016-1025, 2004.

17. M. Morelli and U. Mengali, "An Improved Frequency Offset Estimator for OFDM Applications", IEEE Communications Letters, Vol. 3, No. 3, pp. 75-77, 1999.

18. B. Park, H. Cheon, C. Kang, and D. Hong, "A Novel Timing Estimation Method for OFDM Systems", IEEE Communications Letters, Vol. 7, No. 5, pp. 239-241, 2003.

19. H. Minn, M. Zeng, and V. Bhargava, "On Timing Offset Estimation for OFDM Systems", IEEE Communications Letters, Vol. 4, No. 7, pp. 242-244, 2000.

20. Z. J. Hass and J. Deng, "Dual Busy Tone Multiple Access (dbtma)-a Multiple Access Control Scheme for ad hoc Networks", IEEE Transactions on Communications, Vol. 50, No. 6, pp. 975-985, 2002.

21. S.-L. Wu, Y.-C. Tseng, and J.-P. Sheu, "Intelligent Medium Access for Mobile ad hoc Networks with Busy Tones and Power Control", IEEE Journal on Selected Areas in Communications (JSAC), Vol. 18, No. 9, pp. 1647-1657, 2000.

22. C. Ware, T. Wysocki, and J. Chicharo, "Hidden Terminal Jamming Problems in IEEE 802.11 Mobile ad hoc Networks", Proc. IEEE International Conference on Communications. ICC, Helsinki (Finland), Vol. 1, pp. 261-265, 11-14 June 2001.

23. E. Gelenbe and G. Pujolle, Introduction to Queueing Networks, 2nd edn. plus John Wiley \& Sons, 1995.

24. L. Qian, A. Krishnamurthy, Y. Wang, Y. Tang, P. Dauchy, and A. Onte, "A New Traffi Model and Statistical Admission Control Algorithm for Providing QoS Guarantees to on-line traffic" IEEE Global Telecommunications Conference. GLOBECOM, Vol. 3, No. 29, pp. 1401-1405, 2004.

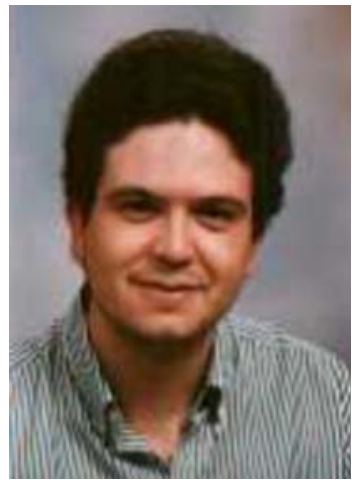

Gil Jiménez, Víctor P. received the B. Eng. in Telecommunications with honors from University of Alcalá in 1998 and the M. Eng. in Telecommunications and the PhD. degree both from the University Carlos III de Madrid in 2001 and 2005, respectively. He is with the Department of Signal Theory and Communications at the University Carlos III de Madrid as an Assistant Professor. He worked at the Spanish Antarctica Base in 1999 as Communications Staff. He visited University of Leeds and Chalmers Technical University in 2003 and 2004 respectively. His research interests include multicarrier communications and signal processing for wireless systems. 


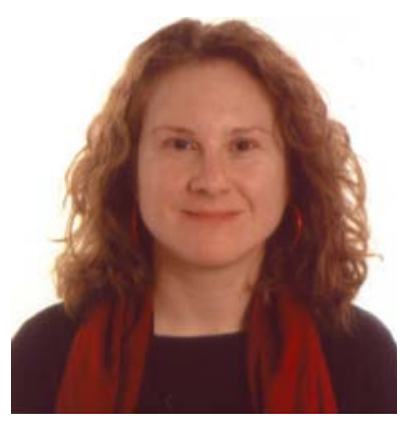

García Armada, Ana received the Telecommunication Engineer degree and the Ph. D in Electrical Engineering both from the Polytechnic University of Madrid (Spain) in 1994 and 1998, respectively. She is currently working as an Associate Professor at the University Carlos III de Madrid, where she has occupied several management positions. She has participated in several national and international research projects, most of them related to OFDM. She is co-author of four books on wireless communications and signal processing. She has published 13 papers in international journals and more than 40 papers in conferences. She has contributed to international organizations such as ITU and ETSI. She has performed research stays in ESA-ESTEC, Kansas University, Stanford University and Bell Labs. Her research interests are simulation of communication systems, multicarrier and MIMO techniques. 